Poster (016)

Gynecologic Pathology, Genetics and Epidemiology

https://doi.org/10.3802/jgo.2021.32.S1.016

\section{Risk reducing salpingo-oophorectomy for germline BRCA mutation carriers: outcomes of single-center experiences in South Korea}

\section{Ok Ju Kang, ${ }^{1}$ Young Jae Lee, ${ }^{2}$ Shin Hwa Lee, ${ }^{1}$ Yong Man Kim ${ }^{1, *}$}

'Asan Medical Center, Seoul, Korea (amcymkim@gmail.com)

${ }^{2}$ Gangneung Asan Hospital, Gangneung, Korea

Objective: This study was aimed to investigate the patient's characteristics and histopathologic findings of risk-reducing salpingo-oophorectomy (RRSO) conducted in Asan Medical Center. Methods: We retrospectively analyzed the medical records of germline BRCA1/BRCA2 mutation carriers who underwent RRSO at Asan Medical Center from January 2009 to December 2020. Results: A total of 274 patients underwent RRSO (136 BRCA1, 135 BRCA2, 3 BRCA1, and 2 mutation carriers). The mean age at the time of surgical intervention was similar for BRCA1 and BRCA2 mutation carriers ( 46.9 and 46.3, respectively). Only 6 surgeries were performed in 2009, while 48 were performed in 2020, with more and more over time. Eight out of 136 (5.8\%) BRCA1 carriers and 9 out of 135 (6.7\%) BRCA2 carriers were unaffected, and others were breast cancer patients. According to the pathologic reports, 6 out of 136 (4.4\%) BRCA1 mutation group were diagnosed with occult malignancy such as tubal cancer, and 4 out of 137 (3.0\%) were pre-malignant lesions, including serous tubal intraepithelial lesion (STIL) or serous tubal intraepithelial carcinoma (STIC). In the BRCA2 mutation group, only 1 out of 135 $(0.7 \%)$ was diagnosed with STIC, and no occult cancer was found. Conclusion: According to our study, germline BRCA mutation carriers tend to go through RRSO in their mid to late 40s, and most of the patients were breast cancer patients. Occult tubal cancer or premalignant lesion (STIC, STIL) were diagnosed more frequently in BRCA1 carriers than BRCA2 carriers.
Shanghai, China (zhuangguanglei@gmail.com)

${ }^{3}$ Shanghai Institute of Organic Chemistry, Chinese Academy of Sciences, Shanghai, China

${ }^{4}$ Shanghai Cancer Institute, Ren Ji Hospital, School of Medicine, Shanghai Jiao Tong University, Shanghai, China

Objective: Müllerian tissue-specific oncogenes, prototyped by PAX8, underlie ovarian tumorigenesis and represent unique molecular vulnerabilities. Further delineating such lineagedependency factors and associated therapeutic implications would provide valuable insights into ovarian cancer biology and treatment. Methods: SOX17 and PAX8 expression were assayed by pan-cancer analysis, single-cell RNA sequencing, immunohistochemistry and immunoblotting. Functional impact of SOX17 or PAX8 depletion was assessed in cell lines and xenografts. The relationship between SOX17 and PAX8 was investigated by immunofluorescence staining, co-immunoprecipitation, RNA sequencing and CUT \& Tag. A highthroughput image-based drug screen was performed, and a novel orally bioavailable cyclin-dependent kinase (CDK) 12/13 covalent antagonist was synthesized to evaluate its anti-tumor efficacy. Results: We identified SOX17 as a new lineage-survival master transcription factor, which shared co-expression pattern with PAX8 in epithelial ovarian carcinoma. Genetic disruption of SOX17 or PAX8 analogously inhibited neoplastic cell viability and downregulated a spectrum of lineage-related transcripts. Mechanistically, we showed that SOX17 physically interacted with PAX8 in cultured cell lines and clinical tumor specimens. The 2 nuclear proteins bound to overlapping genomic regions and regulated a common set of downstream genes, including those involved in cell cycle and tissue morphogenesis. In addition, we revealed that small-molecule inhibitors of transcriptional CDKs effectively reduced SOX17 and PAX8 expression. ZSQ1722, a novel orally bioavailable CDK12/13 covalent antagonist, exerted potent anti-tumor activity in xenograft models.

Conclusion: These findings shed light on an actionable lineagesurvival transcriptional complex in ovarian cancer, and facilitated drug discovery by generating a serial of candidate compounds to pharmacologically target this difficult-to-treat malignancy.

Poster (018)

Epithelial Ovarian Cancer including Borderline Tumor https://doi.org/10.3802/jgo.2021.32.S1.018

\section{Survival analysis of adjuvant chemotherapy impacts in advanced-stage epithelial ovarian cancer}

Waode Radmila, ${ }^{1, *}$ Nugraha Utama Pelupessy, ${ }^{2}$ Isharyah Sunarno ${ }^{3}$

'Department of Obstetrics and Gynecology, Hasanuddin University, Makassar, Indonesia (waode.radmila@gmail.com)

${ }^{2}$ Division of Gynecologic Oncology, Department of Obstetrics and 